\title{
Raman response of Stage-1 graphite intercalation compounds revisited
}

\author{
J.C. Chacón-Torres* and T. Pichlen ${ }^{\dagger}$ \\ Faculty of Physics, University of Vienna, \\ Strudlhofgasse 4, A-1090 Vienna, Austria \\ A.Y. Ganin and M.J. Rosseinsky \\ Department of Chemistry, University of Liverpool, \\ Liverpool L69 7ZD, UK
}

(Dated: October 30, 2018)

\begin{abstract}
We present a detailed in-situ Raman analysis of stage- $1 \mathrm{KC}_{8}, \mathrm{CaC}_{6}$, and $\mathrm{LiC}_{6}$ graphite intercalation compounds (GIC) to unravel their intrinsic finger print. Four main components were found between $1200 \mathrm{~cm}^{-1}$ and $1700 \mathrm{~cm}^{-1}$, and each of them were assigned to a corresponding vibrational mode. From a detailed line shape analysis of the intrinsic Fano-lines of the G- and D-line response we precisely determine the position $\left(\omega_{p h}\right)$, line width $\left(\Gamma_{p h}\right)$ and asymmetry $(q)$ from each component. The comparison to the theoretical calculated line width and position of each component allow us to extract the electron-phonon coupling constant of these compounds. A coupling constant $\lambda_{p h}<0.06$ was obtained. This highlights that Raman active modes alone are not sufficient to explain the superconductivity within the electron-phonon coupling mechanism in $\mathrm{CaC}_{6}$ and $\mathrm{KC}_{8}$.
\end{abstract}

\section{INTRODUCTION}

$\mathrm{Sp}^{2}$ hybridized carbon allotropes have unique structural properties in different dimensions like graphite (3D), graphene (2D), single walled carbon nanotubes (1D), and fullerene molecules ("quasi 0D"). They have been widely studied due to their interesting electronic properties ranging from metallic, semimetallic, (zero gap) semiconducting to wide gap semiconducting and insulating [1-4]. One unique possibility to tailor their electronic properties is by intercalation of alkali and alkaline-earth ions. These intercalation compounds are particular appealing for their application in batteries and because of their superconducting phases. Superconductivity, as a result of alkali-metal intercalation, was first studied by Henning [5] in Graphite Intercalation Compounds (GIC) and further studies [6, 7]. However, until 1981, the critical temperature $\left(\mathrm{T}_{c}\right)$ in stage- $1 \mathrm{XC}_{8} \mathrm{GIC}(\mathrm{X}=\mathrm{K}, \mathrm{Rb}$, and $\mathrm{Cs}$ ) reported was low [1], not higher than $0.135 \mathrm{~K}$ for $\mathrm{CsC}_{8}$, and between $0.39-0.55 \mathrm{~K}$ for $\mathrm{KC}_{8}$. This is surprising since GIC are BCS superconductors based on electron phonon coupling owing an exceptionally high electron phonon coupling constant up to $\lambda=0.45$ in the case of $\mathrm{KC}_{8}$ and high phonon frequency of the optical modes [8]. For instance, using $\lambda=0.45$ and a phonon frequency of $1337 \mathrm{~cm}^{-1}$ a BCS $\mathrm{T}_{c}$ of $\sim 5 \mathrm{~K}$ would be possible in $\mathrm{KC}_{8}$ which is much higher than the observed $\mathrm{T}_{c}$ up to $0.55 \mathrm{~K}$, and this can be related to a screened Coulomb pseudopotential of $\mu^{*}=0.14$, which is on the lower bound with respect to $\mathrm{CaC}_{6}$ [9].

In 1991, the discovery of fullerene intercalation com-

\footnotetext{
* julio.chacon@univie.ac.at Faculty of Physics, University of Vienna, Strudlhofgasse 4, A-1090 Vienna, Austria

$\dagger$ http://epm.univie.ac.at
}

pounds, so-called fullerides, added a new family of organic superconductors of type $\mathrm{A}_{3} \mathrm{C}_{60} \quad(\mathrm{~A}=$ alkali-metal) 10 12. Compared to classical superconductors, and in contrast to $\mathrm{GIC}$ the $\mathrm{T}_{c}$ observed in fullerides is high ranging from $18 \mathrm{~K}$ for $\mathrm{K}_{3} \mathrm{C}_{60}, 28 \mathrm{~K}$ for $\mathrm{Rb}_{3} \mathrm{C}_{60}$, up to $39 \mathrm{~K}$ for $\mathrm{Cs}_{3} \mathrm{C}_{60}[11-13]$. Contrary to GIC, where the highest intercalation level represents the superconducting phase, for fullerides the superconducting phase is a line phase at half filling. Other stable fullerides $\mathrm{A}_{1} \mathrm{C}_{60}, \mathrm{~A}_{4} \mathrm{C}_{60}$ and $\mathrm{A}_{6} \mathrm{C}_{60}$ are either normal metals, Mott-Hubbard insulators or charge transfer insulators [14]. Similar to GIC the superconducting coupling mechanism was described within the framework of BCS theory involving an electron phonon coupling to the intra-molecular modes of $\mathrm{C}_{60}$ 3]. Experimentally, most important for the coupling are the two low energy intra-molecular modes with $H_{g}$ symmetry [15, 16], although theoretically the high frequency phonons have been predicted to play a significant role [17, 18].

For GIC, the observation of superconductivity of $\mathrm{CaC}_{6}$ in 2005 with a high $\mathrm{T}_{c}$ of $11.5 \mathrm{~K}$ [19] triggered further research in the field and led to alternative explanations of the superconducting electron phonon coupling. For instance, Kim et al. attribute superconductivity in $\mathrm{CaC}_{6}$ to the high-energy $\mathrm{C}$ modes [20]. Hinks et al. [21] report that the low-energy modes of the intercalant were responsible for superconductivity inferred from specific heat analysis, while first principle calculations predicts equal coupling to both groups of phonons [9, 22]. Therefore, the exact contribution of the different coupling phonons still remain elusive.

Raman spectroscopy became then an important tool to determine the exact contribution of each phonon, and it opened a route for revealing the coupling mechanism in superconducting fullerides and GICs. Hence, it serves as a key tool to analyze the electron phonon coupling con- 
stant $(\lambda)$ from a renormalization of the optical response of the intra-molecular $\mathrm{C}_{60}$ modes and of the graphitic G-line response. Recent Raman studies on the G-line response of different stage-1 GIC reported the assignment of the electron phonon coupling (EPC) induced line width $\gamma^{E P C}$ to the $1510 \mathrm{~cm}^{-1}$ mode [23 26], which has been explained by the inclusion of non adiabatic phonon calculations [24, 26]. However, the intrinsic G-line response in heavily doped graphite compounds are still elusive because of the influence of defects and laser induced deintercalation, as recently reported using a micro Raman analysis for $\mathrm{CaC}_{6}[24]$ and for $\mathrm{KC}_{8}$ single crystals [27.

In this contribution we report a detailed study of the D- and G-lines in $\mathrm{KC}_{8}, \mathrm{CaC}_{6}$, and $\mathrm{LiC}_{6}$ GIC, in order to unravel their intrinsic phonon components and its relation to the electron phonon coupling constant responsible for superconductivity. From the analysis of the optical phonons observed, we assign their role in the superconductivity coupling mechanism in comparison with previous results of electron doped GIC.

\section{EXPERIMENTAL, AND MEASUREMENT DETAILS}

The synthesis of $\mathrm{KC}_{8}$ was performed in-situ under high vacuum $\left(\sim 4 \times 10^{-8}\right.$ mbar $)$ conditions in a quartz tube with natural graphite flake single crystals from different sources, and a potassium ingot with $99.95 \%$ purity (Aldrich) for the intercalation. Potassium was evaporated until golden crystals were obtained. This phase can be directly assigned to stage- $1 \mathrm{KC}_{8}$ phase from a comparison of the Raman response with previous combined Raman and XRD results [27, 28]. $\mathrm{CaC}_{6}$, and $\mathrm{LiC}_{6}$ were prepared in a sealed ampoule by using a procedure described elsewhere [29]. Highly oriented pyrolytic graphite (HOPG) flakes were degassed and used for lithium and calcium intercalation for 10 days under He atmosphere (ca. $0.5 \mathrm{~atm}$ ). The ampoule was then opened in the glove box and gold colored product was extracted from the melt. Powder x-ray diffraction measurements were carried out using a Stadi-P diffractometer $\left(\mathrm{CuK}_{a}\right)$ to confirm the intercalation stage in $\mathrm{CaC}_{6}$ and $\mathrm{LiC}_{6}$. For the Raman analysis every GIC was kept in vacuum $\left(\sim 4 \times 10^{-8}\right.$ mbar) in order to avoid de-intercalation due to exposure to air. The Raman analysis, was performed with a HORIBA LabRam at room temperature, with a $568 \mathrm{~nm}$ wave length, and $0.25 \mathrm{~mW}$ of laser power. Every spectrum were acquired under the same conditions in a range from $500 \mathrm{~cm}^{-1}$ up to $2500 \mathrm{~cm}^{-1}$ and the line positions were calibrated by gauge lamps.

\section{RESULTS AND DISCUSSION}

In the Raman response of stage-1 GIC eight optical vibrational modes are present [28] in the following irreducible representation:

$$
\Gamma=2 A_{2 u}+2 B_{2 g}+2 E_{1 u}+2 E_{2 g}
$$
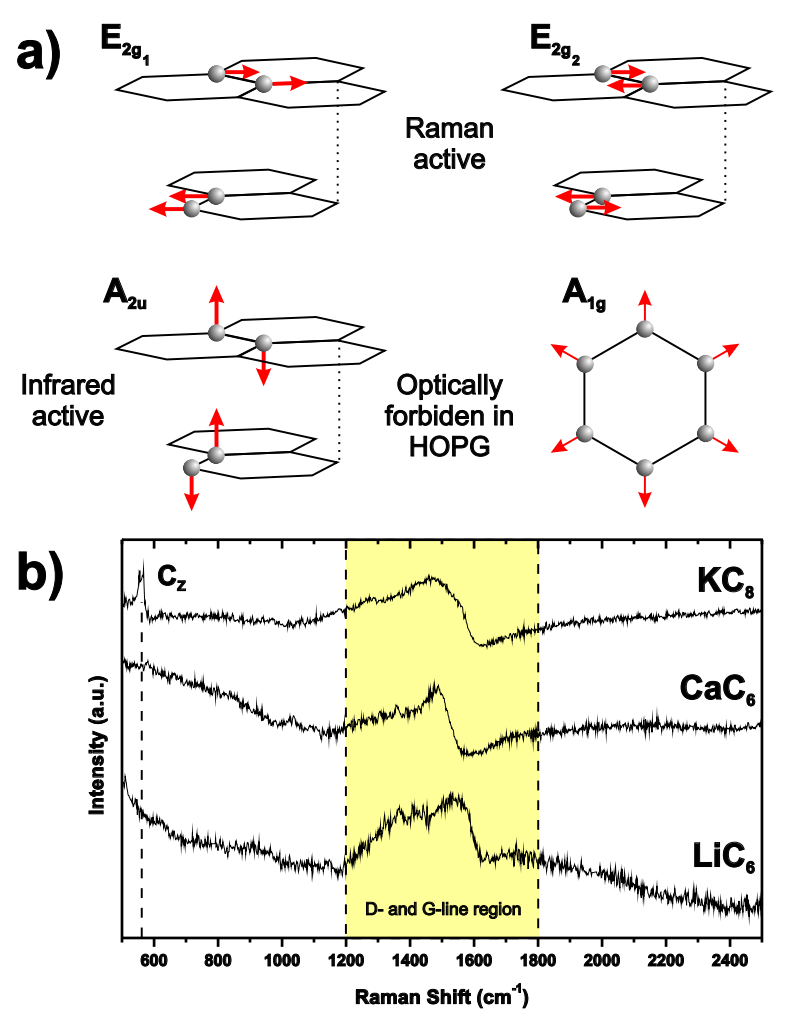

FIG. 1. a) Optical modes of graphite. b) Raman spectra from $\mathrm{KC}_{8}, \mathrm{CaC}_{6}$, and $\mathrm{LiC}_{6}$ taken with $568 \mathrm{~nm}$ laser at room temperature and low laser power of $0.25 \mathrm{~mW}$.

The $E_{2_{g_{1}}}$, and the $E_{2_{g_{2}}}$ vibrational modes are Raman active, and the $A_{2 u}$, and $E_{1 u}$ belong to infra-red active modes [1, 30]. There are some other modes in graphite which are forbidden in perfect graphite and only become active in the presence of disorder like the mode with $A_{1 g}$ symmetry. In Fig. 1 a), the optical modes of graphite are depicted. Previous Raman studies in GIC have confirmed the presence of the $E_{2 g}$ mode around $1600 \mathrm{~cm}^{-1}$, the $A_{2 u}$ (c-axis mode) around $500 \mathrm{~cm}^{-1}$, and the absence of the $A_{1 g}[1,28]$. The $c$-axis mode has being attributed to an out-of-plane $\mathrm{C}$ motion in graphite [24]. This mode correspond to the $\mathbf{M}$ point of the graphene Brillouin zone, and it becomes Raman active when high intercalation levels are achieved. In agreement with the literature, we observe (as shown in in Fig. 1 b), that the c-axis mode is present solely in $\mathrm{KC}_{8}$ around $\sim 560 \mathrm{~cm}^{-1}$. Surprisingly and in agreement with previous studies neither in $\mathrm{CaC}_{6}$, nor in $\mathrm{LiC}_{6}$ this mode is observed [23, 31].

Regarding the G-line response all these previous studies reported one G-line which has a strong Fano line shape due to the coupling and the interference with the conduction electrons. Taking a closer look on the lineshape of the G-line response in Fig. 1 b), one can easily see that more than one component is present, and a detailed line shape analysis is needed in order to unravel their intrinsic response and related electron phonon coupling of these stage-1 GIC. The line-shape analysis of the G-line is discussed in detail below. 
a)

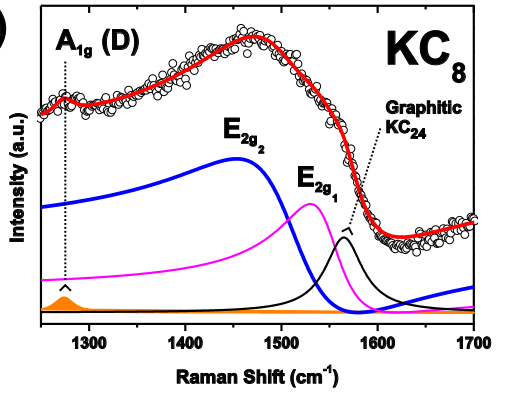

b)

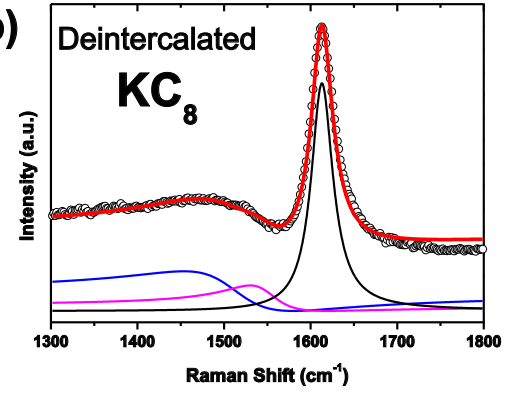

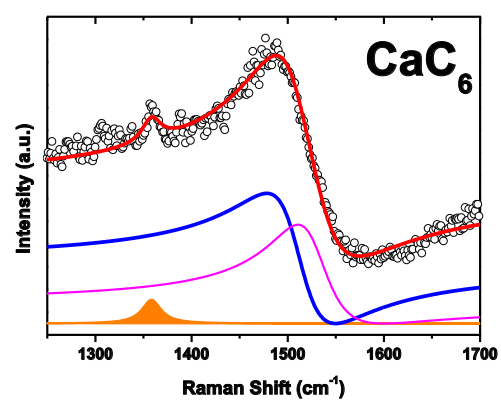
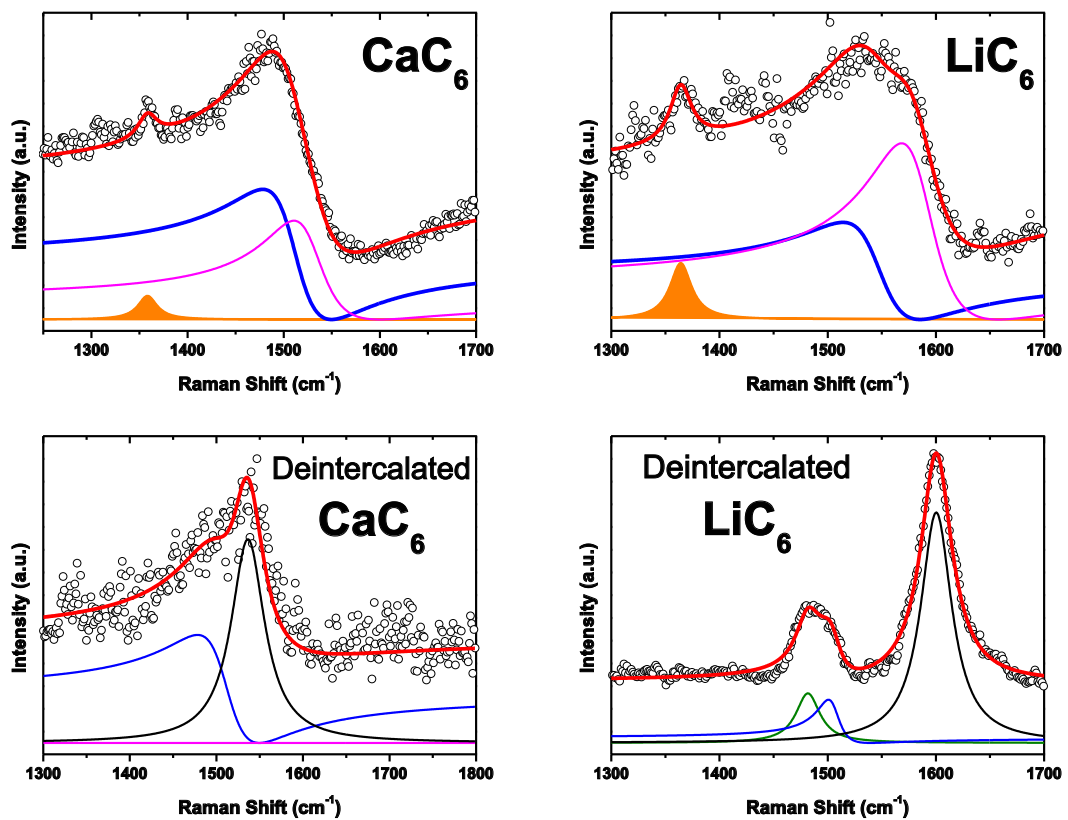

FIG. 2. D- and G-line analysis for stage-1 GIC, and Raman response of their laser induced deintercalated phases. The four

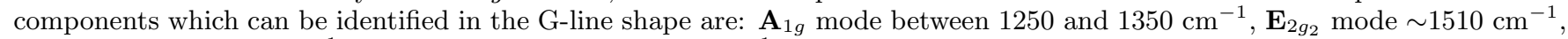
$\mathbf{E}_{2 g_{1}}$ mode at $\sim 1547 \mathrm{~cm}^{-1}$, and stage-2 G-mode $\sim 1560 \mathrm{~cm}^{-1}$. In the upper panel a) we can observe that KC 8 exhibit a strong contribution from the $\mathbf{E}_{2 g_{2}}$ with a broad Fano behavior, which is the finger print for the intrinsic line of stage-1 compound [27]. In the lower panel b) we present the same crystals analyzed in the upper panel but de-intercalated. We can clearly observe the

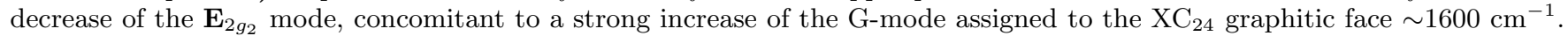

\section{A. Analysis of the intrinsic G-line response of Stage-1 GIC}

The structure of the intercalation stages in graphitic compounds, has been studied and it is well understood from x-ray diffraction [28]. However, the intrinsic Raman response of stage-1 GIC is still complicated by laser induced de-intercalation from a local heating of the sample with different laser power densities [30]. In addition, other factors such as 3D intrinsic disorder of the crystal also strongly affect the Raman response in GIC. For example, a graphite single crystal doped to stage-1 will remain polycrystalline due to a non-homogeneous intercalation. This will limit the achievable doping in these GIC 24, 32].

Hence, the previous experimental and theoretical results on the Raman response of $\mathrm{KC}_{8}$ and $\mathrm{CaC}_{6}$ reported in the literature are not conclusive with respect to the G-line shape and position. In different studies a wide range of different G-line positions between $\sim 1400 \mathrm{~cm}^{-1}$ and $\sim 1600 \mathrm{~cm}^{-1}$ are reported: i.e. at $\sim 1500 \mathrm{~cm}^{-1}[1$, between $1400 \mathrm{~cm}^{-1}$ and $1550 \mathrm{~cm}^{-1}$ [28], $1534 \mathrm{~cm}^{-1}$ [26], $1547 \mathrm{~cm}^{-1}$ [30], $1420 \mathrm{~cm}^{-1}$ and $1582 \mathrm{~cm}^{-1}$ 33]. In more recent experiments for calcium GIC 24, 25], potassium doped graphene and graphite [27, 32], and later in Ligraphite [31], the strongest G-line phonon response is observed around $1510 \mathrm{~cm}^{-1}$ when the sample has the best quality (lowest defect content) and highest intercalation.

In Fig. 2 a) the D- to G-band region of pristine stage1 intercalation compounds with $\mathrm{K}, \mathrm{Ca}$, and $\mathrm{Li}$ is de- picted and clearly shows the presence of shoulders in the response, which indicate different components. Nevertheless, in order to compare with the previous studies [26, 30, 33] we first conducted a line-shape analysis of the G-line by using a single Breit Wigner Fano (BWF) function. This yields parameters which are in good agreement to those results, and confirms that our samples have the same high quality of a true stage-1 compound. This is further supported by the fact that the G-line assigned to stage-2 compounds around $1600 \mathrm{~cm}^{-1}$ is only increased upon e.g. laser induced de-intercalation (see Fig. 2 b).

In a second step a detailed and accurate analysis of the line-shape in the D- to G-band region of these GIC was conducted using four components. The assignment of each component to the $\mathrm{A}_{1 g}(\mathrm{D}), E_{2 g_{2}}, E_{2 g_{1}}$ modes, and the G-line of the stage-2 compound is explained in the following.

Regarding the line-shape, all components have been fitted using BWF functions of the form:

$$
I(w)=I_{0} \frac{\left(1+\frac{w-w_{p h}}{q \Gamma / 2}\right)^{2}}{1+\left(\frac{w-w_{p h}}{\Gamma / 2}\right)^{2}}+A
$$

where $\omega_{p h}$ is the phonon frequency, $\Gamma$ the line width or damping, $\mathbf{q}$ the asymmetry parameter and $\mathrm{A}$ an offset. For the first and fourth peak (D, and $\mathrm{G})$, the asymmetry was $\mathbf{q}=10^{5}$ approaching a Lorentzian function, while the second and third (splitted G-line) have a pronounced Fano interference. In the analysis, in order to get comparable results for each GIC, the same values of $\Gamma$, and 
$\mathbf{q}$ were used to fit each respective component. The parameters are summarized in Table I together with the calculated values from the adiabatic and non-adiabatic phonons from Ref. [26].

The first mode observed in Fig. 2 a) between 1260 and $1360 \mathrm{~cm}^{-1}$ has been previously attributed to particle size effects and/or the presence of disorder [34, 35]. It has been assigned to the $A_{1 g}$ vibration, which is forbidden in perfect graphite. Therefore, this mode is called D-line (intrinsic "defect mediated"), and it involves the contribution from the phonons near the $K$ zone boundary with a Lorentzian line-shape.

The second and third modes observed are assigned to the $E_{2 g}$ graphitic mode of heavily doped graphene layers (Fig. 2 a). Both components have a pronounced asymmetry and they are well described by a BWF lineshape. We label the two modes as $E_{2 g_{1}}$ and $E_{2 g_{2}}$. The $E_{2 g_{1}}$ mode is located between $1528 \mathrm{~cm}^{-1}$ and $1585 \mathrm{~cm}^{-1}$ and it is attributed to not homogeneous or incomplete intercalation in stage-1 compounds [27]. The $E_{2 g_{2}}$ mode locates at $1510 \mathrm{~cm}^{-1}$ for $\mathrm{KC}_{8}$ and $\mathrm{CaC}_{6}$, and $1546 \mathrm{~cm}^{-1}$ for $\mathrm{LiC}_{6}$. It has a clear and strong Fano behavior which is characteristic to the finger print of stage-1 graphite intercalation compounds [24, 27]. When de-intercalation was induced in the samples, a decrease of these $E_{2 g}$ modes was remarkably observed (see Fig. 2 b).

The fourth mode related to the G-line of their respective stage-2 compound is observed at $1612 \mathrm{~cm}^{-1}$ for $\mathrm{KC}_{8}$, $1600 \mathrm{~cm}^{-1}$ for $\mathrm{LiC}_{6}$ and at $1560 \mathrm{~cm}^{-1}$ for $\mathrm{CaC}_{6}$. The surprising low frequency in the case of $\mathrm{CaC}_{6}$ was also found in Ref. [25] and explained as a de-intercalated phase in $\mathrm{CaC}_{6}$. As mentioned above, the increase of this fourth component is highlighted in the partly de-intercalated stage-1 compounds in Fig. 2 b), and points towards a phase separation upon de-intercalation.

\section{B. Analysis of the Electron-Phonon Coupling}

The previous results are very important for the correct determination of the stage, and electron-phonon coupling constant $\lambda_{p h}$ responsible for superconductivity within the BCS theory [24, 26, 36]. This constant is directly related to the intrinsic G-line phonon frequency, and to the adiabatic $\left(\omega_{A}\right)$ and non-adiabatic $\left(\omega_{N A}\right)$ phonon frequencies. Saitta et al. [26] have analyzed the EPC in many different stage-1 GIC from a difference in the experimental phonon frequency to the calculated phonon frequency in the adiabatic and non-adiabatic limit. In order to determine the electron phonon scattering renormalized line width $\gamma^{E P C}$ [24, 26] we used:

$$
\frac{\gamma^{E P C}}{2}=\sqrt{\left(\omega_{p h}-\omega_{A}\right)\left(\omega_{N A}-\omega_{p h}\right)}
$$

We obtain $\gamma^{E P C}$ values for $\mathrm{KC}_{8}, \mathrm{CaC}_{6}$, and $\mathrm{LiC}_{6}$ which are in very good agreement to our experimental $\Gamma_{p h}$ value obtained from our BWF fit, Table II. In Fig. 3 we show the location of our $\gamma^{E P C}$ with respect to the expected linear tendency to $\Gamma_{p h}$ as predicted by Saitta
TABLE I. Fit parameters to the four components of the Dand G-line in the Raman spectra of $\mathrm{KC}_{8}, \mathrm{CaC}_{6}$, and $\mathrm{LiC}_{6}$.

\begin{tabular}{|c|c|c|c|c|c|}
\hline $\mathrm{KC}_{8}$ & $\omega_{p h}\left(\mathrm{~cm}^{-1}\right)$ & $\Gamma_{p h}\left(\mathrm{~cm}^{-1}\right)$ & q & $\omega_{A}{ }^{\mathrm{a}}$ & $\omega_{N A}{ }^{\mathrm{b}}$ \\
\hline $\mathrm{D}$ & 1274 & 24.3 & $10^{5}$ & - & - \\
\hline$E_{2 g_{2}}$ & 1510 & 125.6 & -1.09 & 1223 & 1534 \\
\hline$E_{2 g_{1}}$ & 1547 & 70.9 & -2.02 & 1223 & 1534 \\
\hline $\mathbf{G}^{c}$ & 1565 & 47.0 & $10^{5}$ & - & - \\
\hline $\mathrm{CaC}_{6}$ & $\omega_{p h}\left(\mathrm{~cm}^{-1}\right)$ & $\Gamma_{p h}\left(\mathrm{~cm}^{-1}\right)$ & q & $\omega_{A}{ }^{\mathrm{a}}$ & $\omega_{N A}{ }^{\mathrm{b}}$ \\
\hline $\mathbf{D}$ & 1358 & 24.3 & $10^{5}$ & - & - \\
\hline$E_{2 g_{2}}$ & 1510 & 71.0 & -1.09 & 1446 & 1529 \\
\hline$E_{2 g_{1}}$ & 1528 & 70.9 & -2.02 & 1446 & 1529 \\
\hline $\mathrm{LiC}_{6}$ & $\omega_{p h}\left(\mathrm{~cm}^{-1}\right)$ & $\Gamma_{p h}\left(\mathrm{~cm}^{-1}\right)$ & q & $\omega_{A_{-}^{a}}^{a}$ & $\omega_{N A}{ }^{\mathrm{b}}$ \\
\hline $\mathbf{D}$ & 1364 & 24.3 & $10^{5}$ & - & - \\
\hline$E_{2 g_{2}}$ & 1546 & 71.0 & -1.09 & 1362 & 1580 \\
\hline$E_{2 g_{1}}$ & 1585 & 70.9 & -2.02 & 1362 & 1580 \\
\hline
\end{tabular}

a Calculated Adiabatic $\mathrm{E}_{2 g}$ phonon frequencies Ref. [26] in $\mathrm{cm}^{-1}$.

b Calculated Non-adiabatic $\mathrm{E}_{2 g}$ phonon frequencies Ref. [26] in $\mathrm{cm}^{-1}$.

${ }^{c} \mathrm{G}$-line contribution from $\mathrm{KC}_{24}$ stage-2 compound.

et al. [26]. It is important to notice that some components of the G-line in $\mathrm{KC}_{8}, \mathrm{CaC}_{6}$, and $\mathrm{LiC}_{6}$ bring a $\gamma^{E P C}=0$, which means that they do not show the nonadiabatic effects for layered metals and therefore they do not contribute to the electron-phonon coupling constant $\lambda_{p h}$. In comparison to the experimental $\Gamma^{\exp }$ and $\gamma^{E P C}$ from Ref. [23, 37, 38] (Fig. 3 $\star$ ), our results using the $\mathrm{E}_{2 g_{2}}$ mode are in better agreement to the linear trend expected for $\Gamma \approx \gamma^{E P C}$. This confirms the importance of every optical mode in the range between the adiabatic and non-adiabatic frequency range $\left(\omega_{A}-\omega_{N A}\right)$, and confirms that the $\mathrm{E}_{2 g_{2}}$ component is the intrinsic stage-1 vibrational mode with the strongest non-adiabatic effect on the EPC.

We now turn to a detailed analysis of the EPC constant $\lambda_{p h}$. Different values have been already reported and used to calculate the critical temperature of $\mathrm{KC}_{8}, \mathrm{CaC}_{6}$, and $\mathrm{LiC}_{6}$ with values around $5 \mathrm{~K}, 11.5 \mathrm{~K}$, and $0.9 \mathrm{~K}$, respectively, in agreement with some experimental and theoretical studies [8, 40]. In order to extract $\lambda_{p h}$ from the phonon line-width $(\Gamma)$ and position $\left(\omega_{p h}\right)$ from our Raman data we used [41]:

$$
\lambda_{\Gamma, K}=\frac{A_{u c} F_{\Gamma, K}^{2}}{2 M \omega_{\Gamma, K} v_{F}^{2}}
$$

where the electron-phonon coupling strength is given by $D_{\exp }$ :

$$
\Delta \Gamma_{G}=\frac{A_{u c} D_{e x p}^{2}}{8 M v_{F}^{2}}
$$

and $A_{u c}$ is defined as the area of the graphene unit cell, $M$ is the carbon atom mass, $v_{F}$ is the Fermi velocity, $\Delta \Gamma_{G}$ is the Landau damping phonon decay rate given by 


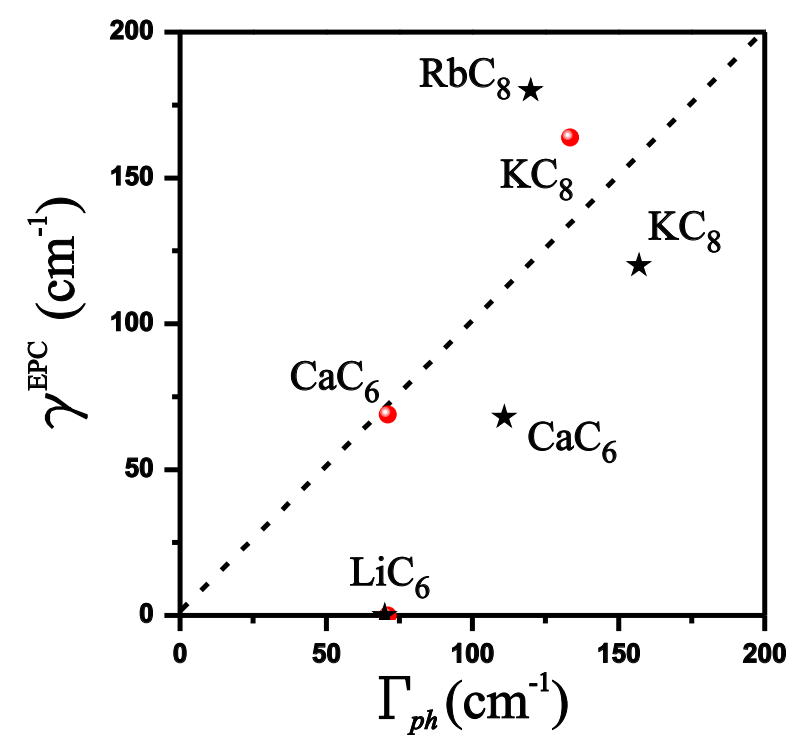

FIG. 3. Calculated $\gamma^{E P C}$ (Eq. 1) for different GIC as function of their width $\Gamma_{p h}$. Black stars $(\star)$ correspond to experimental values from Ref. 23, 37, 38]. The dashed line represents the approximation of $\Gamma_{p h} \approx \gamma^{E P C}$. The red dots show our calculated EPC, which are in better agreement to the expected approximation to the $\Gamma_{p h}$ values.

$\Delta \Gamma_{G}=\Gamma_{p h}-\Gamma_{G r a p h i t e}$, and $F_{\Gamma, K}^{2}$ has dimensionality of a force taking in consideration the lattice displacement along the corresponding optical phonon mode. By using Eq. 2 and the definition of $F_{\Gamma}^{2}=4\left\langle D_{\Gamma}^{2}\right\rangle_{F}$, and $F_{K}^{2}=$ $2\left\langle D_{K}^{2}\right\rangle_{F}$ from Ref. [39, 41] we calculate the values for $\lambda_{\Gamma, K}$ for each phonon in the $\Gamma-K$ branch observed in the G-line region as summarized in the right column of Table II. $\left\langle D_{\Gamma, K}^{2}\right\rangle_{F}$ were taken from the $\mathrm{DFT}_{G G A}$ calculations in Graphite [39] as they are closer to our electron-phonon coupling strength $\left(D_{\text {exp }}\right)$.

By using the averaged electron-phonon coupling constant $\lambda_{p h}=\lambda_{\Gamma}+\lambda_{K}$, and the position $\omega_{p h}$ from the strongest optical mode in $\mathrm{KC}_{8}, \mathrm{CaC}_{6}$, and $\mathrm{LiC}_{6}$ one can estimate the critical temperature $\mathrm{T}_{c}$ using McMillan's formula [42]. Taking our $\omega_{p h}$ values converted in to phonon temperature $\Theta, \mu^{*} \approx 0.14$ from [43], and $\lambda_{p h}$ from the Raman analysis, we obtain $\lambda_{p h}<0.06$ values, which are too low to explain superconductivity within EPC mechanism using these high-frequency Raman active modes.

However, this is not a general behavior in intercalation compounds. Electron-phonon studies in alkaliintercalated fullerenes showed the possibility to attribute the strongest $\lambda_{p h}$ contribution for superconductivity to the $\mathrm{Hg}(1)$ mode in $\mathrm{A}_{3} \mathrm{C}_{60}$ fullerides [15, 16]. More over, in agreement to the analysis reported by Yao et al. in Ref. [16] our $D_{\text {exp }}$ presented the same trend as the one observed in fullerides intercalation compounds. Therefore, we can confirm that the larger the value of $1 / \mathbf{q}$, the weaker the coupling strength $D_{\text {exp }}$ in GIC and fullerides.

On the other hand, in comparison to the EPC constant
TABLE II. Electron-phonon coupling parameters from the G-line Raman analysis. The values of $\omega_{p h}, \Gamma_{p h}, \gamma^{E P C}$ are in $\mathrm{cm}^{-1}$ and they were extracted from the BWF analysis of the Raman spectrum. $D_{\text {exp }}$ is the electron-phonon coupling strength from Eq. 3 in $(\mathrm{eV} / \AA)$.

\begin{tabular}{cccccccc}
\hline \hline $\mathrm{KC}_{8}$ & $\omega_{p h}$ & $\Gamma_{p h}$ & $\gamma^{E P C}$ & $\gamma^{E P C \mathrm{a}}$ & $D_{\exp }$ & $O B^{\mathrm{b}}$ & $\lambda_{K, \Gamma^{\mathrm{c}}}$ \\
\hline $\mathbf{D}$ & 1274 & 24.3 & 230 & - & 14 & $K$ & 0.024 \\
$E_{2 g_{2}}$ & 1510 & 125.6 & 163 & 157 & 51 & $\Gamma$ & 0.020 \\
$E_{2 g_{1}}$ & 1547 & 70.9 & 0 & - & 36 & $\Gamma$ & - \\
$\lambda_{p h}$ & & & & & & & 0.044 \\
& & & & & & & \\
\hline $\mathrm{CaC}_{6}$ & $\omega_{p h}$ & $\Gamma_{p h}$ & $\gamma^{E P C}$ & $\gamma^{E P C \mathrm{a}}$ & $D_{\exp }$ & $O B^{\mathrm{b}}$ & $\lambda_{K, \Gamma^{\mathrm{c}}}$ \\
\hline $\mathbf{D}$ & 1358 & 24.3 & 0 & - & 14 & $K$ & 0.022 \\
$E_{2 g_{2}}$ & 1510 & 71.0 & 68 & 68 & 36 & $\Gamma$ & 0.020 \\
$E_{2 g_{1}}$ & 1525 & 70.9 & 34 & 36 & 36 & $\Gamma$ & 0.019 \\
$\lambda_{p h}$ & & & & & & & 0.061 \\
& & & & & & & \\
\hline $\mathrm{LiC}_{6}$ & $\omega_{p h}$ & $\Gamma_{p h}$ & $\gamma^{E P C}$ & $\gamma^{E P C \mathrm{a}}$ & $D_{\exp }$ & $O B^{\mathrm{b}}$ & $\lambda_{K, \Gamma}{ }^{\mathrm{c}}$ \\
\hline $\mathbf{D}$ & 1364 & 24.3 & 43 & - & 14 & $K$ & 0.022 \\
$E_{2 g_{2}}$ & 1546 & 71.0 & 157 & - & 36 & $\Gamma$ & 0.019 \\
$E_{2 g_{1}}$ & 1585 & 70.9 & 0 & 0 & 36 & $\Gamma$ & - \\
$\lambda_{p h}$ & & & & & & & 0.041 \\
\hline \hline
\end{tabular}

a Calculated phonon full line width at half maximum due to phonon decay in dressed electron-hole pairs $\gamma_{\sigma}^{E P C}$ Ref. [26].

b Optical branch assignment based in [8, 39].

c Electron-phonon coupling constant from Eq. 2

$\lambda_{A R P E S}$ reported using an analysis of the self energy results in ARPES [8, 44], our $\lambda_{p h}$ values are about a factor of 10-15 lower. Since, in the case of $\mathrm{CaC}_{6}$ superconductivity was confirmed at $\mathrm{T}_{c}=11.5 \mathrm{~K}$, only the $\lambda_{A R P E S}$ [4 would be sufficient to explain this high superconducting transition temperature. Hence, the low $\lambda_{p h}$ proves that optical modes from the G-line in stage-1 GIC are not sufficient to explain $\mathrm{T}_{c}$ in the electron-phonon driven superconducting coupling mechanism and additional not optically active modes might play an important role.

\section{CONCLUSIONS}

We have performed a detailed in-situ Raman study of the most common $\mathrm{GIC}\left(\mathrm{KC}_{8}, \mathrm{CaC}_{6}\right.$, and $\left.\mathrm{LiC}_{6}\right)$. We identify four main peaks in the $\mathrm{D}$ - to G-band region, and all these Raman responses match the spread of different line shapes reported in the literatures so far. From an evaluation of the fine structure in the G-line response we assign each peak to their corresponding vibrational mode and phonon branch.

We found the strongest Fano behavior of the G-line at $1510 \mathrm{~cm}^{-1}$ in $\mathrm{KC}_{8}$ and $\mathrm{CaC}_{6}$, not like in $\mathrm{LiC}_{6}$, which highlights the importance of this mode to the superconductivity coupling mechanism within the BSC theory, and confirms the importance of this $E_{2 g_{2}}$ mode to nonadiabatic effects. By using this mode, we obtain a very good agreement to the theoretical predicted line-width $\gamma^{E P C} \simeq \Gamma_{p h}$ especially for $\mathrm{CaC}_{6}$.

Finally, we find a very small EPC $\lambda_{p h}<0.06$ which is much too low to explain the high $\mathrm{T}_{c}$ in this graphite 
intercalated compounds. This points out that, other phonons including acoustic modes and other electronic states might play an important role in explaining the superconducting pairing in GIC.

\section{ACKNOWLEDGMENTS}

We acknowledge for the financial support of the project FWF-I377-N16, the OEAD AMADEUS PROGRAM financing, and the comments from Dr. Hidetsugu Shiozawa and Dr. Christian Kramberger. AG and MJR thank EPSRC for funding.
[1] M. S. Dresselhaus and G. Dresselhaus, Advances In Physics 30, 139 (1981)

[2] A. Jorio, G. Dresselhaus, and M. S. Dresselhaus, Carbon Nanotubes. Advanced Topics in the Synthesis, Structure, Properties and Applications(2008)

[3] O. Gunnarsson, Reviews of Modern Physics 69, 575 (1997)

[4] A. H. Castro Neto, F. Guinea, N. M. R. Peres, K. S. Novoselov, and A. K. Geim, Reviews of Modern Physics 81, 109 (2009)

[5] G. Hennig and L. Meyer, Physical Review 87, 439 (1952)

[6] N. B. Hannay, T. H. Geballe, B. T. Matthias, K. Andres, P. Schmidt, and D. Macnair, Physical Review Letters 14, 225 (1965)

[7] M. Kobayashi and I. Tsujikawa, Journal of the Physical Society of Japan 46, 1945 (1979)

[8] A. Gruneis, C. Attaccalite, A. Rubio, D. V. Vyalikh, S. L. Molodtsov, J. Fink, R. Follath, W. Eberhardt, B. Buchner, and T. Pichler, Physical Review B 79, 205106 (2009)

[9] M. Calandra and F. Mauri, Physical Review Letters 95, 237002 (2005)

[10] R. M. Fleming, A. P. Ramirez, M. J. Rosseinsky, D. W. Murphy, R. C. Haddon, S. M. Zahurak, and A. V. Makhija, Nature 352, 787 (1991)

[11] A. F. Hebard, M. J. Rosseinsky, R. C. Haddon, D. W. Murphy, S. H. Glarum, T. T. M. Palstra, A. P. Ramirez, and A. R. Kortan, Nature 350, 600 (1991)

[12] M. J. Rosseinsky, A. P. Ramirez, S. H. Glarum, D. W. Murphy, R. C. Haddon, A. F. Hebard, T. T. M. Palstra, A. R. Kortan, S. M. Zahurak, and A. V. Makhija, Physical Review Letters 66, 2830 (1991)

[13] A. Y. Ganin, Y. Takabayashi, Y. Z. Khimyak, S. Margadonna, A. Tamai, M. J. Rosseinsky, and K. Prassides, Nature Materials 7, 367 (2008)

[14] M. Capone, M. Fabrizio, C. Castellani, and E. Tosatti, Reviews of Modern Physics 81, 943 (2009)

[15] J. Winter and H. Kuzmany, Physical Review B 53, 655 (1996)

[16] M. Yao, V. Pischedda, and A. San Miguel, Journal of Physics-Condensed Matter 23, 115701 (2011)

[17] C. M. Varma, J. Zaanen, and K. Raghavachari, Science 254, 989 (1991)

[18] M. Schlüter, M. Lannoo, M. Needels, G. A. Baraff, and D. Tomanek, Journal of Physics and Chemistry of Solids 53, 1473 (1992)

[19] T. E. Weller, M. Ellerby, S. S. Saxena, R. P. Smith, and N. T. Skipper, Nature Physics 1, 39 (2005)

[20] J. S. Kim, R. K. Kremer, L. Boeri, and F. S. Razavi,
Physical Review Letters 96, 217002 (2006)

[21] D. G. Hinks, D. Rosenmann, H. Claus, M. S. Bailey, and J. D. Jorgensen, Physical Review B 75, 014509 (2007)

[22] I. I. Mazin, L. Boeri, O. V. Dolgov, A. A. Golubov, G. B. Bachelet, M. Giantomassi, and O. K. Andersen, Physica C - Superconductivity and its Applications 460, 116 (2007)

[23] J. Hlinka, I. Gregora, J. Pokorny, C. Herold, N. Emery, J. F. Mareche, and P. Lagrange, Physical Review B 76, 144512 (2007)

[24] M. P. M. Dean, C. A. Howard, S. S. Saxena, and M. Ellerby, Physical Review B 81, 045405 (2010)

[25] A. Mialitsin, J. S. Kim, R. K. Kremer, and G. Blumberg, Physical Review B 79, 064503 (2009)

[26] A. M. Saitta, M. Lazzeri, M. Calandra, and F. Mauri, Physical Review Letters 100, 226401 (2008)

[27] J. C. Chacón-Torres and T. Pichler, Physica Status Solidi b 248, 2744 (2011)

[28] S. A. Solin and N. Caswell, Journal of Raman Spectroscopy 10, 129 (1981)

[29] N. Emery, C. Herold, M. d'Astuto, V. Garcia, C. Bellin, J. F. Mareche, P. Lagrange, and G. Loupias, Physical Review Letters 95, 087003 (2005)

[30] R. J. Nemanich, S. A. Solin, and D. Guerard, Physical Review B 16, 2965 (1977)

[31] G. L. Doll, P. C. Eklund, and J. E. Fischer, Physical Review B 36, 4940 (1987)

[32] C. A. Howard, M. P. M. Dean, and F. Withers, Physical Review B 84, 241404 (2011)

[33] P. C. Eklund and K. R. Subbaswamy, Physical Review B 20, 5157 (1979)

[34] F. Tuinstra and J. L. Koenig, Journal of Chemical Physics 53, 1126 (1970)

[35] A. C. Ferrari and J. Robertson, Physical Review B 61, 14095 (2000)

[36] L. Pietronero and S. Strässler, Europhysics Letters 18, 627 (1992)

[37] D. Guerard and A. Herold, Carbon 13, 337 (1975)

[38] G. L. Doll, M. H. Yang, and P. C. Eklund, Physical Review B 35, 9790 (1987)

[39] M. Lazzeri, C. Attaccalite, L. Wirtz, and F. Mauri, Physical Review B 78, 081406 (2008)

[40] G. Profeta, M. Calandra, and F. Mauri, Nature Physics 8, 1 (2012)

[41] D. M. Basko, S. Piscanec, and A. C. Ferrari, Physical Review B 80, 165413 (2009)

[42] M. Schlüter, M. Lannoo, M. Needels, G. A. Baraff, and D. Tomanek, Physical Review Letters 68, 526 (1992)

[43] W. L. McMillan, Physical Review 167, 331 (1968)

[44] T. Valla and Z. Pan, Physics and Applications of Graphene (InTech, 2011) 